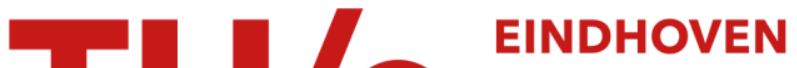 UNIVERSITY OF TECHNOLOGY
}

\section{Data-driven modeling of impedance biosensors}

\section{Citation for published version (APA):}

Ramírez-Chavarría, R. G., Alvarez-Serna, B. E., Schoukens, M., \& Alvarez-lcaza, L. (2021). Data-driven modeling of impedance biosensors: A subspace approach. Measurement Science and Technology, 32(10), [104009]. https://doi.org/10.1088/1361-6501/ac0b15

\section{Document license: \\ TAVERNE}

DOI:

10.1088/1361-6501/ac0b15

Document status and date:

Published: 01/10/2021

\section{Document Version:}

Publisher's PDF, also known as Version of Record (includes final page, issue and volume numbers)

\section{Please check the document version of this publication:}

- A submitted manuscript is the version of the article upon submission and before peer-review. There can be important differences between the submitted version and the official published version of record. People interested in the research are advised to contact the author for the final version of the publication, or visit the $\mathrm{DOI}$ to the publisher's website.

- The final author version and the galley proof are versions of the publication after peer review.

- The final published version features the final layout of the paper including the volume, issue and page numbers.

Link to publication

\section{General rights}

Copyright and moral rights for the publications made accessible in the public portal are retained by the authors and/or other copyright owners and it is a condition of accessing publications that users recognise and abide by the legal requirements associated with these rights.

- Users may download and print one copy of any publication from the public portal for the purpose of private study or research.

- You may not further distribute the material or use it for any profit-making activity or commercial gain

- You may freely distribute the URL identifying the publication in the public portal.

If the publication is distributed under the terms of Article 25fa of the Dutch Copyright Act, indicated by the "Taverne" license above, please follow below link for the End User Agreement:

www.tue.nl/taverne

Take down policy

If you believe that this document breaches copyright please contact us at:

openaccess@tue.nl

providing details and we will investigate your claim. 


\section{PAPER}

Data-driven modeling of impedance biosensors: a subspace approach

To cite this article: Roberto G Ramírez-Chavarría et al 2021 Meas. Sci. Technol. 32104009

View the article online for updates and enhancements. 


\title{
Data-driven modeling of impedance biosensors: a subspace approach
}

\author{
Roberto G Ramírez-Chavarría $^{1, *}\left(\mathbb{D}\right.$, Bryan E Alvarez-Serna $^{1}$, Maarten Schoukens ${ }^{2}$ (D) \\ and Luis Alvarez-Icaza ${ }^{1}$ (D)
}

${ }^{1}$ Instituto de Ingeniería, Universidad Nacional Autónoma de México, 04510 Ciudad de México, México

${ }^{2}$ Control Systems Group, Eindhoven University of Technology, 5600 MB Eindhoven, The Netherlands

E-mail: RRamirezC@iingen.unam.mx

Received 28 February 2021, revised 7 June 2021

Accepted for publication 14 June 2021

Published 28 June 2021

\begin{abstract}
A data-driven scheme for modeling electrical impedance in biosensors is presented by a subspace method working with the singular value decomposition of structured voltage and current data. Contrary to the classical electrical impedance spectroscopy (EIS) methods, our scheme uses simple instrumentation, works in time-domain, provides fast results, and does not require semi-empirical assumptions to retrieve structured models from data. We show how data-driven models exhibit a close relationship with lumped-element circuits, encoding dielectric and conductive properties detected by the sensor in the range from $10 \mathrm{kHz}$ up to $10 \mathrm{MHz}$. Performance results are shown for calibration networks and two case studies: (i) a buffer solution, and (ii) a biological cell suspension. Finally, the viability of the scheme is discussed when compared with the classical EIS method.
\end{abstract}

Keywords: biosensors, subspace methods, impedance spectroscopy, data-driven models, signal processing

(Some figures may appear in colour only in the online journal)

\section{Introduction}

The analysis of biological media through biosensors and electrical impedance spectroscopy (EIS) is, nowadays, an attractive label-free method for understanding bio-electrical processes and characterizing the frequency dependence of dielectric and conductive phenomena [1,2]. Owing to their high sensitivity and versatility, impedance biosensors are ubiquitous in several applications to electrically characterize biological media such as tissues [3], bacteria [4], cells [5, 6] and nucleic acids [7], to mention only a few. Once EIS is experimentally determined, its analysis involves data fitting to a user-defined lumped-parameter equivalent circuit model (ECM) [8]. This traditional workflow has some inherent limitations, mainly, the choice of a correct model structure able to capture the impedance dynamics and to provide physical

\footnotetext{
* Author to whom any correspondence should be addressed.
}

meaning to the parameters. To this end, one should have prior knowledge $(\mathrm{PK})$ of the sample under study to select an adequate model, otherwise, either over- or under-fitting issues may limit the accuracy of the method [9].

Currently, several investigations have contributed with novel techniques to circumvent inherent limitations in EIS measurement and modeling. For instance, in [10] the authors implement a data-analytic modeling approach, able to decode electrical impedance measurements of different cell lines by using a polynomial regression. An impedance modeling method based on a genetic algorithm is presented in [11] to identify the most suitable model by solving an optimization problem. For speeding-up estimation results, in [12] a quadratic interpolation retrieves the parameters of a lumpedparameter network using embedded hardware. Also, in [13] introduces learning-based models to quantitatively relate the impedance parameters with bacterial concentrations at high reliability. More recently, [14] presents an impedancebased method for sensing biological cell suspensions which 
correlates a distribution function with impedance measurements to determine the concentration. In summary, trends in advanced optimization techniques and data-driven methods offer a flexible way to circumvent the limitations of the classical biosensors models [15].

On the other hand, an ECM has structural and dynamical properties which, under some conditions, can be described by a linear-time-invariant model [16-18]. For instance, a statespace (SS) model of bioimpedance for characterizing hematocrit is presented in [19]. In [20], the authors propose an infinite-impulse-response model that relates the parameters of an ECM for bioimpedance applications. Moreover, an autoregressive model for impedance measurements in micro-scale applications was introduced in [21]. However, the dynamical point-of-view for bioimpedance measurements has been barely explored, and its applications remain limited. One of the main drawbacks is how to obtain an adequate model that, on the one hand, captures the whole dynamics of the system with enough sensitivity; and on the other, relates the model parameters with an ECM to provide physical interpretation.

Nowadays, the availability of data from impedance measurements together with advanced processing techniques pave the road to develop novel data-driven methods to construct models describing impedance data in a robust way. In this work, we introduce, to the best of our knowledge, a novel scheme to estimate a model describing the underlying dynamics of impedance-based biosensors. The proposal works around the predictor-based subspace identification (PBSID) method, which estimates from time-domain data, an SS model and a transfer function (TF) encoding the parameters of an ECM. The subspace approach has three main contributions. (i) It uses simple instrumentation based on minimal hardware design and robust signal processing techniques instead of analog circuits for signal conditioning and transformation to retrieve impedance spectra. (ii) The proposal provides fast results compared to the classical EIS since our technique operates with broadband signals and non-iterative processing methods instead of sweeping the frequency of a single sine stimulus and time-consuming procedures for data fitting. (iii) The subspace method does not require semi-empirical assumptions to retrieve structured models from data. For all cases, we show the performance and limitations of the method in calibration networks and two case studies, which are compared with the classical EIS method, to assess the viability of our proposal.

The rest of the paper is organized as follows. Section 2 introduces theoretical basis of impedance measurements and the subspace modeling method. The details of the measurement setup and the sensor are presented in section 3. Experimental results for calibration networks and two case studies are shown in section 4 along a thorough discussion. Finally, section 5 is devoted to the conclusions derived from this work.

\section{Theoretical framework}

\subsection{Principle of impedance biosensors}

2.1.1. Electrical impedance spectroscopy. An impedancebased biosensor is commonly studied in a wide frequency (a)

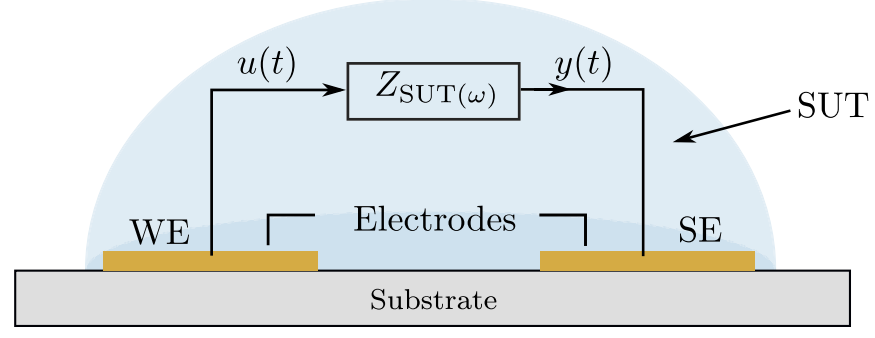

(b)

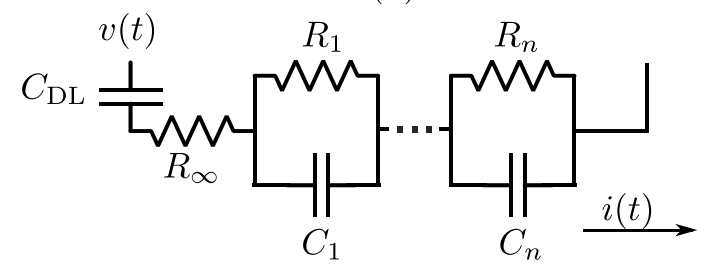

Figure 1. Working principle of impedance biosensors. (a) Sketch of the impedance measurement for a SUT. (b) Generalized ECM of the impedance $Z_{\mathrm{ECM}}(\omega)$ describing the SUT.

range using EIS as follows. The biosensor is excited with a small-amplitude alternating signal $u(t)$, which produces a linear output $y(t)$ [22], i.e. the sample does not produce overtones [23]. Typically, the former is a voltage $v(t)$, whereas the latter is the current flowing to through the sample $i(t)$. By taking the discrete Fourier transform $\mathcal{F}$ of such a signals, $I\left(\omega_{i}\right):=\mathcal{F}\{i(t)\}$ and $V\left(\omega_{i}\right):=\mathcal{F}\{v(t)\}$, the measured impedance spectrum is given by

$$
Z\left(j \omega_{i}\right):=\frac{V\left(\omega_{i}\right)}{I\left(\omega_{i}\right)}=|Z|(\cos (\phi)+j \sin (\phi)),
$$

at an arbitrary frequency $\omega_{i}$, where $j=\sqrt{-1},|Z|$ is the impedance magnitude, and $\phi$ is the phase angle between the phasors, for $i=1,2, \ldots, N$ frequency points.

2.1.2. Non-Faradaic (NF) impedance biosensors. In figure 1(a) we depict a simplified sketch of the working principle when measuring NF impedance in biosensors. It works in the frequency range from $\mathrm{kHz}$ up to tens of $\mathrm{MHz}$, where dielectric effects such as interfacial and relaxation phenomena dominate [24]. Meanwhile, diffusion effects modeled by the Warburg impedance can be disregarded due to the absence of a redox couple and the relatively high-frequency range of study [25]. A working electrode (WE) provides the excitation signal $u(t)$ to the sample under test (SUT) and the sensing electrode (SE) detects the electrical response $y(t)$ of the sample. Under some conditions [26], the SUT has a specific linear impedance $Z_{\mathrm{SUT}}(\omega)$, which depends on the angular frequency $\left(\omega_{i}\right)$, as well as on the physical properties of the sample, such as size, concentration, permittivity and conductivity [26].

2.1.3. Electrical circuit model (ECM). The ECM is one of the most common models to describe the response of an impedance-based biosensor [27]. A classical representation 
of an ECM for NF impedance measurements is shown in figure $1(\mathrm{~b})$, with an equivalent impedance $Z_{\mathrm{ECM}}(\omega) \in \mathbb{C}$ given by

$$
Z_{\mathrm{ECM}}(\omega)=R_{\infty}+\frac{1}{j \omega C_{\mathrm{DL}}}+\sum_{i=1}^{n} \frac{R_{i}}{1+j \omega \tau_{i}},
$$

such that $Z_{\mathrm{ECM}}(\omega) \approx Z_{\mathrm{SUT}}(\omega)$. Without loss of generality, $R_{\infty}$ is the high-frequency dependent resistance, $C_{\mathrm{DL}}$ is the electrode-electrolyte double layer capacitance, and $\tau_{i}=R_{i} C_{i}$ is the $i$ th time-constant associated to the effective capacitance $C_{i}$ and resistance $R_{i}$ of an $i$ th process detected by the sensor. Albeit the impedance model (2) provides a simplistic way to describe the properties of the SUT, in practice, the capacitors $C_{\mathrm{DL}}$ and $C_{\mathrm{i}}$ may have a non-ideal behavior and should be reformulated as constant-phase elements (CPEs) [28] whose impedance is

$$
Z_{\mathrm{CPE}}=\frac{1}{(j \omega)^{\alpha} Q}
$$

where $Q$ is the non-ideal capacitance and $\alpha \in(0,1]$ is a fractional exponent tunning the non-ideality. Note that, if $\alpha=1$ the CPE resembles an ideal capacitor. However, to avoid numerical complexity when dealing with fractional-order models as (3), throughout this work we assume an imperceptible contribution of CPE and use ideal capacitors instead [29]. Though this may not provide accurate fitting results as CPE do, it preserves physical meaning and yields a well-posed approximation to prove the data-driven performance of the PBSID technique.

\subsection{PBSID for impedance biosensors}

Let us consider that the dynamics of an electrical impedance biosensor described by the parametric model $Z_{\mathrm{ECM}}(\omega)$, can also be modeled by the following SS model written in the predictor form [30] as

$\mathcal{S}_{\mathrm{p}}:= \begin{cases}x_{k+1} & =\tilde{A} x_{k}+\tilde{B} z_{k}, \\ y_{k} & =C x_{k}+D u_{k}+e_{k},\end{cases}$

which elements are given by $\tilde{A}=A-K C, \tilde{B}=[B-K D, K]$ and $z_{k}=\left[u_{k}^{\top}, y_{k}^{\top}\right]^{\top}$. In the above SS model, $k$ is a discretetime instant, $u_{k} \in \mathbb{R}$ and $y_{k} \in \mathbb{R}$ are the input and output sampled sequences of $u(t)$ and $y(t)$, respectively, $x_{k} \in \mathbb{R}^{n}$ is the predicted state and $e_{k}$ is the innovation sequence. $A \in \mathbb{R}^{n \times n}$, $B \in \mathbb{R}^{n}, C \in \mathbb{R}^{n}, D \in \mathbb{R}$ and $K \in \mathbb{R}^{n}$ are the state, input, output, direct feed-through and Kalman gain matrices, respectively.

The problem addressed in this work is to provide a modeling scheme which (semi)-automatically estimates a SS model for impedance biosensors using the PBSID algorithm [31, 32]. The proposed approach can be summarized in the following steps.

2.2.1. Step 1: vector autoregressive with exogenous inputs (VARX) model estimation. Given an impedance measurement as shown in figure 1(a), the available set of measurements is given by $\left\{\left(u_{k}, y_{k}\right) \mid k=0,1,2, \ldots, N\right\}$, where $u_{k}=u\left(t=k / f_{s}\right)$ and $y_{k}=y\left(t=k / f_{s}\right)$ are the discrete versions, sampled at $f_{s}$, of the input voltage and output current, respectively. Define $p \in \mathbb{N}^{+}$and $f \in \mathbb{N}^{+}$as the past and future data windows, respectively, with $f=p$. By iterative substituting (4) at an initial time $k=k-p$, the predicted state $x_{k} \in \mathbb{R}^{n}$ is

$$
x_{k}=\tilde{A}^{p} x_{k-p}+\mathcal{K} z_{k-p},
$$

where $\quad z_{k-p}=\left[u_{k-p, p}, y_{k-p, p}\right]^{\top}$ given by $u_{k-p, p}=$ $\left[u_{k}, \ldots, u_{k-p}\right]$ and $y_{k-p, p}=\left[y_{k-1}, \ldots, y_{k-p}\right]$, and $\mathcal{K} \in \mathbb{R}^{n \times p}$ is the extended controllability matrix

$$
\mathcal{K}=\left[\begin{array}{llll}
\tilde{A}^{p-1} \tilde{B} & \tilde{A}^{p-2} \tilde{B} & \cdots & \tilde{B}
\end{array}\right],
$$

with $\tilde{A} \in \mathbb{R}^{n \times n}, \tilde{B} \in \mathbb{R}^{n}$ and $p$ the past window. Moreover, it is known that: (i) impedance-based biosensors operate in openloop, (ii) $Z_{\mathrm{ECM}}(\omega)$ is stable, and (iii) $\tilde{A}^{j}=0$ is nilpotent with degree $p$ for all $j \geqslant p$ [33]. Then, $\tilde{A}^{p} \approx 0$, such that (6) simplifies as

$$
x_{k} \approx \mathcal{K} z_{k-p}
$$

From (5) and (8), it follows that

$$
y_{k} \approx \mathcal{M}_{p} z_{k-p}+D u_{k}+e_{k}
$$

where $\mathcal{M}_{p} \in \mathbb{R}^{(p+f)}$ is the set of Markov parameters given by

$$
\mathcal{M}_{p}=C \mathcal{K}=\left[\begin{array}{llll}
C \tilde{A}^{p-1} \tilde{B} & C \tilde{A}^{p-2} \tilde{B} & \cdots & C \tilde{B}
\end{array}\right] .
$$

Hence, (9) corresponds to a VARX model structure, for which the optimal set $\mathcal{M}_{p}$ can be estimated by solving the following least-squares problem

$$
\mathcal{M}_{p}^{\star}=\min _{\Theta}\left\|Y_{k}-\Theta_{p} \mathcal{D}_{k-p, p}\right\|_{F}^{2}
$$

where $\mathcal{D}_{k-p, p} \in \mathbb{R}^{(p+f) \times(N-p)}$ is the input/output data matrix $\left[\begin{array}{lll}z_{k-1} & z_{k-2} \ldots & z_{k-p}\end{array}\right], Y_{k} \in \mathbb{R}^{(N-p)}$ is the predicted output vector $\left[y_{p}, \ldots, y_{N}\right]$, and $\|\cdot\|_{F}$ denotes the Frobenius norm. Interestingly, the problem in (11) has a closed-form solution involving only algebraic operations.

2.2.2. Step 2: SS model estimation. Once the VARX was estimated, the next step is to estimate the number of states $n$, such that it will be possible to estimate the matrices $A, B, C$ and $D$ describing the dynamics of an impedance-based biosensor.

Now, we introduce the future horizon $f=p$ to define the extended observability matrix $\Gamma \in \mathbb{R}^{(f \times n)}$ as

$$
\Gamma=\left[\begin{array}{c}
C \\
C \tilde{A} \\
\vdots \\
C \tilde{A}^{f-1}
\end{array}\right]
$$


By multiplying the approximation in (8) by $\Gamma$, we have

$$
\Gamma \mathcal{K}=\left[\begin{array}{cccc}
C \tilde{A}^{p-1} \tilde{B} & C \tilde{A}^{p-2} \tilde{B} & \cdots & C \tilde{B} \\
0 & C \tilde{A}^{p-1} \tilde{B} & \ddots & C \tilde{A} \tilde{B} \\
\vdots & \ddots & \ddots & \vdots \\
0 & \cdots & 0 & C \tilde{A}^{p-1} \tilde{B}
\end{array}\right]
$$

which is an upper block triangular matrix. By inspection, one can approximate the matrix (13) with the optimal Markov parameters retrieved in (11). The approximated matrix $\widehat{\Gamma \mathcal{K}} \in$ $\mathbb{R}^{p \times(p+f)}$ is given by

$[\widehat{\Gamma \mathcal{K}}]_{i, j}= \begin{cases}{\left[\mathcal{M}_{p}^{\star}\right]_{i, j}} & \text { for } i \leqslant j \\ 0 & \text { for } i>j\end{cases}$

with $i=1, \ldots, p$ and $j=1, \ldots,(p+f)$. Then, it is possible to formulate the following problem

$$
\Gamma \hat{X}_{k}=\widehat{\Gamma \mathcal{K}} \mathcal{D}_{k-p, p}
$$

given a matrix of state-sequences $\hat{X}_{k} \in \mathbb{R}^{n \times(N-p)}$ and the data matrix $\mathcal{D}_{k-p, p} \in \mathbb{R}^{(p+f) \times(N-p)}$. Thereby, the problem is to estimate the state-sequence $\hat{X}_{k}$ by computing the row space of $\widehat{\Gamma K} \mathcal{D}_{k-p, p}$ via singular value decomposition (SVD) [34],

$$
\widehat{\Gamma \mathcal{K}} \mathcal{D}_{k-p, p}=U \Sigma V^{\top}
$$

where $U \in \mathbb{R}^{p \times p}$ and $V \in \mathbb{R}^{(N-p) \times(N-p)}$ are orthonormal matrices, and $\Sigma \in \mathbb{R}^{p \times(N-p)}$ is a diagonal matrix of singular values. As the main result, SVD retrieves a vector $\tilde{\Sigma} \in \mathbb{R}^{p}$ containing the following useful information.

- The significant singular values of $\tilde{\Sigma}$ represent the number of states of the ECM.

- The number of states correspond to the optimal rank of the SVD [35], as well as an estimate of the ECM order, i.e. $\hat{n}$.

Such remarks allow us to to avoid over-parametrization and also to reduce the SS model complexity [36]. Thus, the estimated state-sequence $\hat{X}_{k}$ is straightforward computed as

$$
\hat{X}_{k, \hat{n}}=\Sigma_{\hat{n}}^{1 / 2} V_{\hat{n}}^{\top}
$$

where $\hat{X}_{k, \hat{n}} \in \mathbb{R}^{\hat{n}}$ is the truncated SS sequence, $\Sigma_{\hat{n}} \in \mathbb{R}^{\hat{n}}$ is a diagonal matrix with the significant singular values and $V_{\hat{n}} \in$ $\mathbb{R}^{\hat{n} \times(N-p)}$ is a matrix of significant vectors.

Once the state-sequence, as well as the output and input signals are known, the system matrices $A, B, C, D$ and $K$ are estimated by finding the optimal solution, in a least-squares sense, of the following linear problems

$$
\begin{gathered}
\hat{\mathcal{Q}}=\min _{\mathcal{Q}}\left\|\hat{X}_{k, \hat{n}}-\mathcal{Q P}\right\|_{F}^{2}, \\
\hat{\mathcal{R}}=\min _{\mathcal{R}}\left\|Y_{k}-\mathcal{R} \mathcal{T}\right\|_{F}^{2},
\end{gathered}
$$

where $\mathcal{Q}=\left[\begin{array}{ll}\tilde{A} & \tilde{B}\end{array}\right], \mathcal{P}=\left[\begin{array}{ll}\hat{X}_{k, \hat{n}} & \mathcal{D}_{k-p, p}\end{array}\right]^{\top}, \mathcal{R}=\left[\begin{array}{ll}C & D\end{array}\right]$ and $\mathcal{T}=\left[\begin{array}{ll}\hat{X}_{k, \hat{n}} & u_{k-p, p}\end{array}\right]^{\top}$. Once $\hat{\mathcal{Q}}$ and $\hat{\mathcal{R}}$ are estimated, the system matrices are straightforward given by

$$
\begin{gathered}
\hat{\tilde{A}}=\hat{A}+\hat{K} \hat{C}, \\
\hat{\tilde{B}}=\left[\begin{array}{ll}
\hat{B}-\hat{K} \hat{D} & \hat{K}
\end{array}\right],
\end{gathered}
$$

and introducing the notation $\{i\}$ for indexing the $i$ th column block of a matrix, we have that $\hat{\mathcal{R}}\{1\}=\hat{C}, \hat{\mathcal{R}}\{2\}=\hat{D}$, $\hat{\tilde{B}}\{1\}=\hat{B}-\hat{K} \hat{D}, \hat{\tilde{B}}\{2\}=\hat{K}$, such that $\hat{B}=\hat{\tilde{B}}\{1\}+\hat{K} \hat{D}$ and $\hat{A}=\hat{\tilde{A}}+\hat{K} \hat{C}$. As a result, we can obtain a minimal realization of a SS model describing the dynamics of impedance-based biosensors

2.2.3. Step 3: TF approximation. The estimated SS model relates the ECM of figure 1(b) by considering that the system states are the voltage drops in each R-C pair, for $i=1, \ldots, n$, and in the double-layer capacitance, such that $x_{k}=\left[\begin{array}{llll}v_{1} & \cdots & v_{n} & v_{\mathrm{DL}}\end{array}\right]^{\top}$. Thereby, with the estimates $\hat{A}, \hat{B}, \hat{C}$ and $\hat{D}$, the PBSID-associated TF is $\hat{H}_{\mathrm{PBSID}}(q)=$ $\hat{C}(q I-\hat{A})^{-1} \hat{B}+\hat{D}$, where $I$ the identity matrix and $q$ is the forward shift operator. The TF $\hat{H}_{\mathrm{PBSID}}(q)$ is then converted to its continuous-time version, $\hat{H}_{c}(s)$, by a bilinear transformation [37], such that $\hat{H}_{c}(s)$ encodes the parameters of an ECM (see figure 1(b)) as

$$
\hat{H}_{c}(s ; \boldsymbol{\theta})=\hat{d}+\frac{\hat{b}_{\mathrm{DL}}}{s}+\sum_{i=1}^{\hat{n}} \frac{\hat{b}_{i}}{s+\hat{a}_{i}},
$$

with $\boldsymbol{\theta}=\left[\hat{b}_{1} \hat{b}_{2} \cdots \hat{b}_{n} \hat{a}_{1} \hat{a}_{2} \cdots \hat{a}_{n} \hat{b}_{\mathrm{DL}} \hat{d}\right]$, whose elements are given by equating (2) and (23) as follows

$$
\hat{d}=R_{\infty}, \quad \hat{b}_{\mathrm{DL}}=\frac{1}{C_{\mathrm{DL}}}, \quad \hat{b}_{i}=\frac{1}{C_{\mathrm{i}}}, \quad \hat{a}_{i}=\frac{1}{\tau_{i}}, \quad \frac{\hat{b}_{i}}{\hat{a}_{i}}=R_{i} .
$$

Hence, one is able to estimate the circuit parameter by simple algebraic manipulations PBSID-based model and it does not require iterative methods for data fitting as in classical EIS [38].

2.2.4. Step 4: frequency response computation. As the last step, the frequency response (FR) of the model (23) is computed. Let consider $s=j \omega_{k}$, where $\omega_{k}$ is the $k$ th frequency point of the FR such that an impedance spectrum $Z_{\mathrm{PBSID}}\left(j \omega_{k}\right)$ can be computed as

$$
Z_{\mathrm{PBSID}}\left(j \omega_{k}\right)=\hat{H}_{c}\left(j \omega_{k}\right) \approx \hat{C}\left(j \omega_{k} I-\hat{A}\right)^{-1} \hat{B}+\hat{D},
$$

for $\omega_{k}=\left\{\omega_{1}, \omega_{2}, \ldots, \omega_{F}\right\}$ a set of excited frequencies within the band of interest. Interestingly, by using the FR, one is able 
DAS

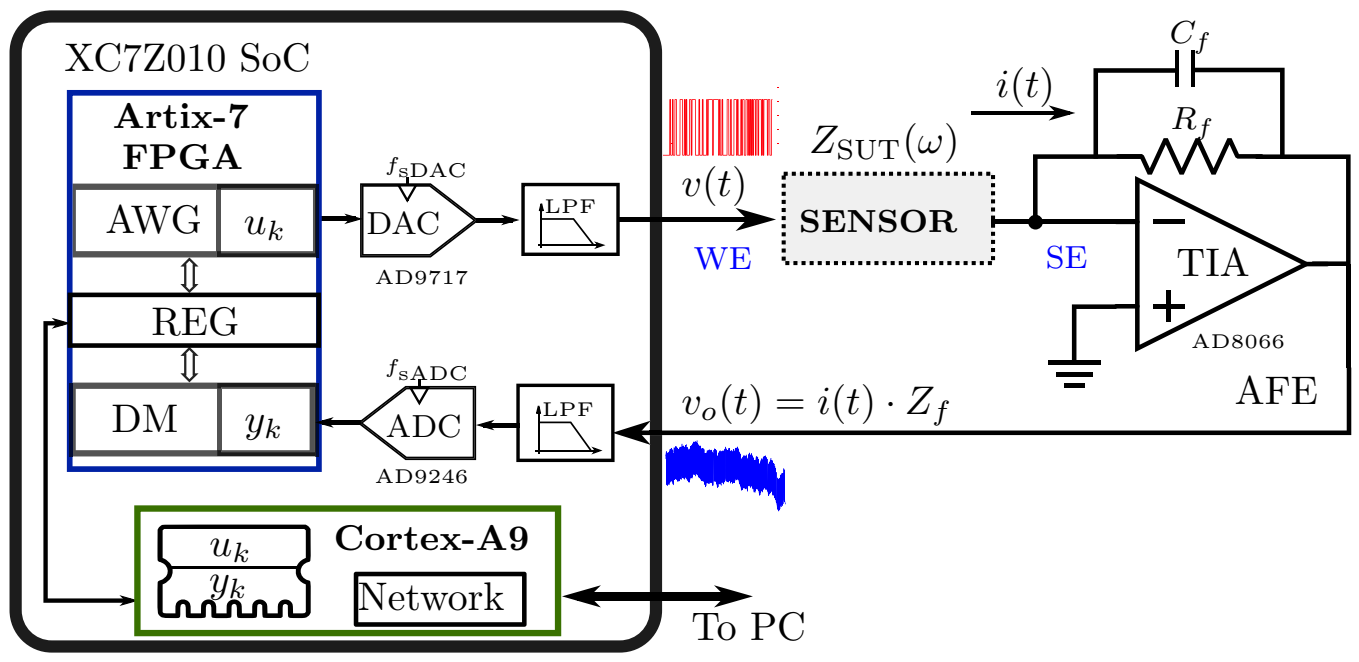

Figure 2. Schematic diagram of the measurement device for measuring input/output signals from impedance biosensors.

to numerically obtain an EIS-like description, thus avoiding large measurement times, bench-top instruments and semiempirical procedures [39], and consequently, the estimated model has both time- and frequency-domain interpretation.

\section{Experimental}

\subsection{Measurement device}

The schematic diagram of the measurement device is shown in figure 2 and fully described in [21]. It is based on a customdesigned data acquisition system based on a XC7Z010 systemon-chip alongside a dedicated analog-front-end (AFE). An Artix-7 field-programmable gate array (FPGA) operates for signal generation and acquisition, whereas the AFE performs operations for signal conditioning and conversion. The input sequence $u_{k}$ is generated by an AD9717 14-bit digital to analog converter (DAC) at a sampling rate $f_{\mathrm{sDAC}}=20 \mathrm{MHz}$ of an arbitrary waveform generator (AWG) module within the FPGA. The analog output $v(t)$ is applied to the WE of the sensor, which detects the impedance $Z_{\text {SUT }}(\omega)$. On the other hand, a low-noise transimpedance amplifier (TIA), built around an AD8066 chip, monitors the current $i(t)$ flowing through the sensor at the SE via the feedback impedance $Z_{f}=R_{f} / / C_{f}$. The output of the TIA is then proportional to the current as $v_{o}(t)=i(t) \cdot Z_{f}$. Afterward, the output from the AFE is synchronously digitized by the digital module with an AD9246 14-bit analog to digital converter operating at a sampling rate $f_{\mathrm{sADC}}=100 \mathrm{MHz}$ to produce the output sequence $y_{k}$. Once the signals $u_{k}$ and $y_{k}$ are collected into a register (REG), the FPGA streams them to a 32-bit dual-core ARM Cortex-A9 processor. This latter runs an application for transferring the the input/output sequences to a personal computer where the PBSID algorithm is implemented.

3.1.1. Identification input signal. The PBSID method described in section 2.2, as other identification methods, must

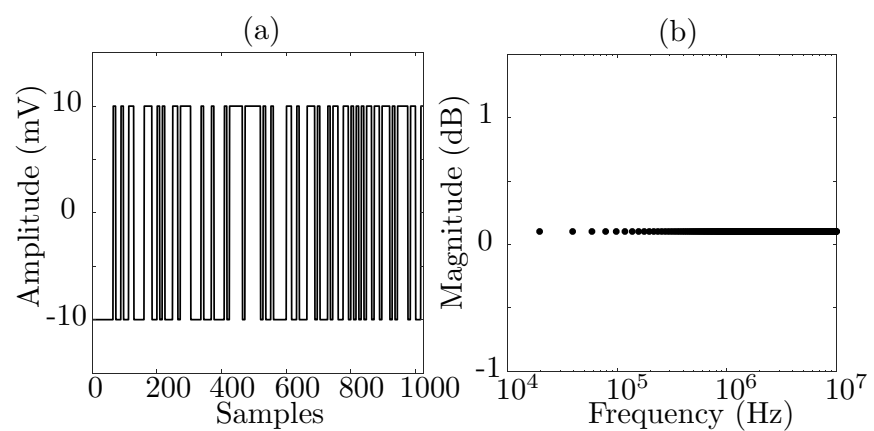

Figure 3. PRBS excitation for impedance biosensors.

(a) Time-domain signal of order $m=10$ and length $M=1023$ samples. (b) Frequency-domain magnitude spectrum in the range from $19.5 \mathrm{kHz}$ up to $10 \mathrm{MHz}$.

work under persistence of excitation (PE) [40] to excite all the modes of the system to provide consistent estimates of the model parameters. Even though in classical EIS, a sine wave is used as the excitation signal since it easy to generate, it does not satisfies the PE condition. Thereby, we use a pseudo random binary signal (PRBS) as the input signal $v(t)$ for the impedance biosensor.

In this work, the spectral content of the PRBS is bandlimited in the frequency range of $10^{4}-10^{7} \mathrm{MHz}$, to minimize the low-frequency diffusion and double-layer effects [41], and for accessing conductive and dielectric phenomena such as interfacial and relaxation processes [42]. Following this rationale, we designed a PRBS of order $m=10$ with 1023 samples exciting the band from $f_{\min }=f_{\mathrm{sDAC}} / 2^{m}=19.5 \mathrm{kHz}$ to $f_{\max }=f_{\mathrm{sDAC}} / 2=10 \mathrm{MHz}$, determined by the DAC sampling frequency $f_{\mathrm{sDAC}}=20 \mathrm{MHz}$. Figure 3 (a) shows the designed PRBS in the time-domain, with an amplitude switching between $\pm 10 \mathrm{mV}$ to work within the impedance linear region. The frequency-domain representation of the PRBS is shown in figure 3(b) showing a flat magnitude spectrum covering the frequency range of interest [43]. 


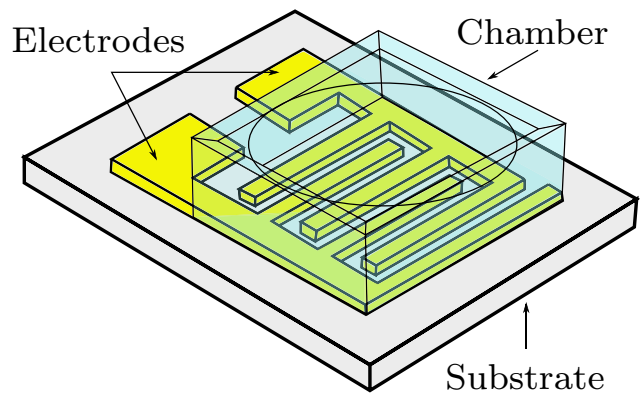

Figure 4. Sketch of the impedance sensor with interdigitated structure (not-to-scale).

3.1.2. Impedance sensor. The sensing element for bioimpedance applications is shown in figure 4. The sensor has a pair of gold micron-sized interdigital electrodes (IDEs) fabricated over a glass substrate with $10 \times 6.5 \times 0.4 \mathrm{~mm}$ dimensions. In this work, the IDE-based biosensor comprises 100 bands of $W=10 \mu \mathrm{m}$ wide, separated by a distance $G=$ $10 \mu \mathrm{m}$, with a band length of $L=1600 \mu \mathrm{m}$. The experimental arrangement considers a polymeric chamber covering the IDE sensitive area and bonded to the substrate, meanwhile the contact pads of the electrodes are exposed to connect them to the measurement system as shown in figure 2 . The plastic chamber allows to better control the experiments for working with a sample volume of $10 \mu \mathrm{l}$ dropped over the IDE surface using a pipette.

\section{Results and discussion}

We present the results for two calibration networks with different dynamics to illustrate the versatility of our proposal. Moreover, the subspace modeling approach is applied in two experimental studies: a buffer solution and a biological cell culture. We test the performance of the PBSID scheme in the following scenarios. Firstly, the time-domain estimation accuracy is evaluated by the variance-accounted-for (VAF) given by

$$
\mathrm{VAF}_{k}=\left\{1-\frac{\operatorname{var}\left(y-\hat{y}_{\text {PBSID }}\right)}{\operatorname{var}(y)}\right\} \cdot 100 \%
$$

where $\hat{y}_{\text {PBSID }}$ and $y$ are the estimated and the measured output sequences, respectively, and $\operatorname{var}(\cdot)$ denotes the variance. On the other hand, we obtain the FR of the PBSID-based model to compare it with the ideal impedance, for the calibration cases, and with classical EIS model, for the practical study cases. Similarly to (26), we use the frequency-domain VAF to determine the accuracy as

$$
\operatorname{VAF}_{\omega}=\left\{1-\frac{\operatorname{var}\left(Z-Z_{\text {PBSID }}\right)}{\operatorname{var}(Z)}\right\} \cdot 100 \%
$$

with $Z$ the ideal or true impedance, and $Z_{\mathrm{PBSID}}$ the impedance model retrieved by the subspace scheme. Note that (27) should be evaluated for the impedance magnitude and phase, separately. Finally, to asses the parametric estimation performance we compute the deviation among the retrieved parameters as follows

$$
\delta_{\mathrm{p}}=\left|\frac{\theta_{\mathrm{T}}-\theta_{\mathrm{PBSID}}}{\theta_{\mathrm{T}}}\right| \cdot 100 \%,
$$

where $\theta_{\mathrm{T}}$ corresponds to the true parameter given by the exact value of the calibration network or by the classical EIS method, meanwhile $\theta_{\mathrm{PBSID}}$ is the estimated parameter with the PBSID scheme.

\subsection{Calibration networks}

The proposed PBSID scheme was calibrated using two electrical networks constructed with passive discrete components with $\pm 1 \%$ and $\pm 5 \%$ tolerance, for resistors and capacitors, respectively. The true value of such a components was measured with a UNI-T ${ }^{\mathrm{TM}}$ UT612 commercial LCR meter (error $<1 \%$ ). For these experiments, we considered $R=100$ repetitions to account for measurement variability. The time-domain $\mathrm{VAF}_{k}$ was computed for each repetition and then averaged over $R$. Then, the model parameters of the PBSID-based TF in (23) were compared with the nominal values to test the accuracy of our proposal. Ultimately, the frequency-domain $\mathrm{VAF}_{\omega}$ was computed using the mean impedance spectra for the model in (25) and the ideal impedance in (2).

4.1.1. Single dispersion circuit. To test the subspace-based modeling, we start with simplest case, namely a single dispersion circuit with one time-constant [12]. The calibration network has the structure shown in the inset of figure 5(a), whose components have the values $\theta_{\mathrm{T}}$ listed in table 1 . In figure 5(a), we show the SVD plot for ten singular values, where only the first is significant due to its magnitude is, on average, four orders greater than that of the other singular values. Thus, the magnitude gap between the singular values 1 and 2 means that $\hat{n}=1$ corresponds to the model order estimate. By inspection of the circuit structure, the first-order model has sense, owing to the network has one pole provided by the time-constant $\tau_{1}=R_{1} C_{1}$. As a result, the time-domain accuracy of the PBSID-based model was $\mathrm{VAF}_{k}=99.5 \%$ for the estimated output sequence. On the other hand, table 1 summarizes the results for the single dispersion circuit, including the PBSID estimates $\theta_{\text {PBSID }}$ alongside their uncertainty $s_{\mathrm{p}}$, as well as the deviation $\% \delta_{\mathrm{p}}$ among the mean estimates by PBSID and the true values $\theta_{\mathrm{T}}$. Results show that the worst case was for the resistor $R_{\infty}$ with an error of $2.25 \%$ which could be related to the high-frequency artifacts; meanwhile, for the other three parameters, the error was less than $0.30 \%$, thus indicating enough accuracy of the data-driven model. As the last step, figure 5(b) shows the FR of both the ideal impedance $Z_{\mathrm{ECM}}$ (continuous line) and the FR of the PBSID model $Z_{\mathrm{PBSID}}$ (dashed line). The frequency-domain accuracy was determined with a $\mathrm{VAF}_{\omega}$ of $99.6 \%$ for the magnitude and $99.4 \%$ for the phase, with a maximum error of $\approx 4.5 \Omega$ and $\approx 0.63^{\circ}$, respectively. This test verifies that the data-driven modeling 


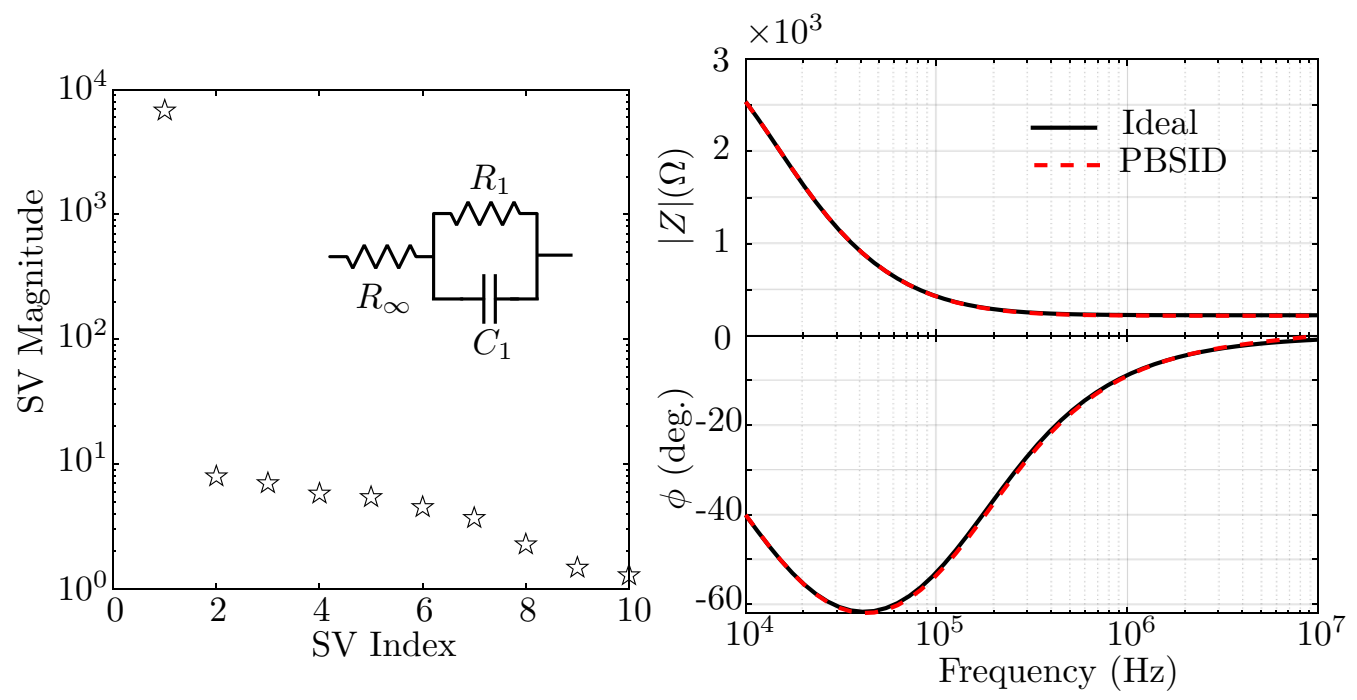

Figure 5. Subspace modeling of a single-dispersion calibration network. (a) Singular value plot showing the estimated model order $\hat{n}=1$ using the PBSID-approach. Inset: structure of the first-order measured circuit. (b) FR magnitude and phase spectra in the range from $10 \mathrm{kHz}$ up to $10 \mathrm{MHz}$.

Table 1. Estimated parameters via PBSID modeling of a single dispersion circuit.

\begin{tabular}{lrrr}
\hline Parameter (units) & \multicolumn{1}{c}{$\theta_{\mathrm{T}}$} & \multicolumn{1}{c}{$\theta_{\mathrm{PBSID}} \pm s_{\mathrm{p}}$} & $\% \delta_{\mathrm{p}}$ \\
\hline$R_{\infty}(\Omega)$ & 221.50 & $216.51 \pm 0.15$ & 2.25 \\
$\tau_{1}(\mu \mathrm{s})$ & 15.15 & $15.17 \pm 0.27$ & 0.13 \\
$C_{1}(\mathrm{nF})$ & 4.62 & $4.63 \pm 0.03$ & 0.21 \\
$R_{1}(\mathrm{k} \Omega)$ & 3.28 & $3.27 \pm 0.04$ & 0.30 \\
\hline
\end{tabular}

scheme provides an adequate model order and structure, and the FR can be regarded as an EIS-like experiment.

4.1.2. Multiple dispersion circuit. The second network for calibration has a multiple dispersion structure, namely three time-constants as shown in the inset of figure 6(a). The true values of the circuit elements are listed in the second column of table 2. In figure 6(a) we show the singular value plot for the calibration network. Interestingly, the SVD plot depicts that the first three singular values are those significant since their large magnitude reflects useful information of the network dynamics; whereas, the indexes of singular values above 4 do not contribute to the circuit behavior. This rationale provides, therefore, an estimate $\hat{n}=3$ of the model order. It can be confirmed given that the circuit corresponds to a third-order model given by the three time-constants $\tau_{i}$ for $i=1,2,3$. Afterwards, the accuracy of the PBSID-based model was determined with a $\mathrm{VAF}_{k}=99.4 \%$, thus indicating that the model well described the time-domain data. In table 2, we show the results for the parametric estimation via PBSID, and the error, $\% \delta_{\mathrm{p}}$, among the mean estimates and the true values of the circuit elements. Interestingly, for all the parameters the accuracy is above $98.37 \%$, with maxima errors of $1.17 \%$ for the capacitor $C_{2}$ and $1.63 \%$ for the time-constant $\tau_{3}$. On the other hand, figure 6(b) depicts the ideal impedance $Z_{\mathrm{ECM}}$ (continuous line) and the FR of the PBSID model $Z_{\mathrm{PBSID}}$ (dashed line). The frequency-domain validation shows the largest error in frequencies below $10^{5} \mathrm{~Hz}$, leading to a $\mathrm{VAF}_{\omega}$ of $99.5 \%$ and $98.7 \%$ for the magnitude and phase, respectively. Finally, this experiment verifies that the proposed PBSID scheme has enough sensitivity and resolution to identify relatively high-order models commonly encountered in bio-impedance applications [3].

\subsection{Case study experiments}

Once we validated the subspace approach on synthetic experiments, we modeled the impedance data of two media with interesting characteristics in biosensors field. These experiments were repeated $R=10$ times to guarantee reproducibilty and to statistically analyze the estimation results. Furthermore, we compared the FR of the data-driven model with the classical EIS, measured directly after our proposal using a singlesine sweep method in the range from $10^{4} \mathrm{~Hz}$ up to $10^{7} \mathrm{~Hz}$. Finally, we fitted the measured data to the same model structure but considering CPE instead of ideal capacitors using the nonlinear least-squares method and the Levenberg-Marquardt algorithm, as in the classical EIS.

4.2.1. Phospate buffer saline (PBS). As the first experiment, we measured PBS, an isotonic buffer commonly used in biological applications. The solution was prepared to obtain $1 \mathrm{X}$ PBS of $\mathrm{pH} 7.4$, with conductivity $\sigma_{\mathrm{PBS}}=1.6 \mathrm{~S} \mathrm{~m}^{-1}$ and relative permittivity $\epsilon_{\mathrm{PBS}}=78$. A droplet of $50 \mu \mathrm{l}$ was placed over the electrodes of the sensor as shown in figure 4 and as described in section 3 .

Figure 7(a) shows the SVD plot for ten values, where one can see that the singular values $\{1,2\}$ are those significant. Hence, the magnitude gap, between the singular values 2 and 3 , provides an estimate model order of $\hat{n}=2$. Following the PBSID workflow, the accuracy of the SS second-order model 

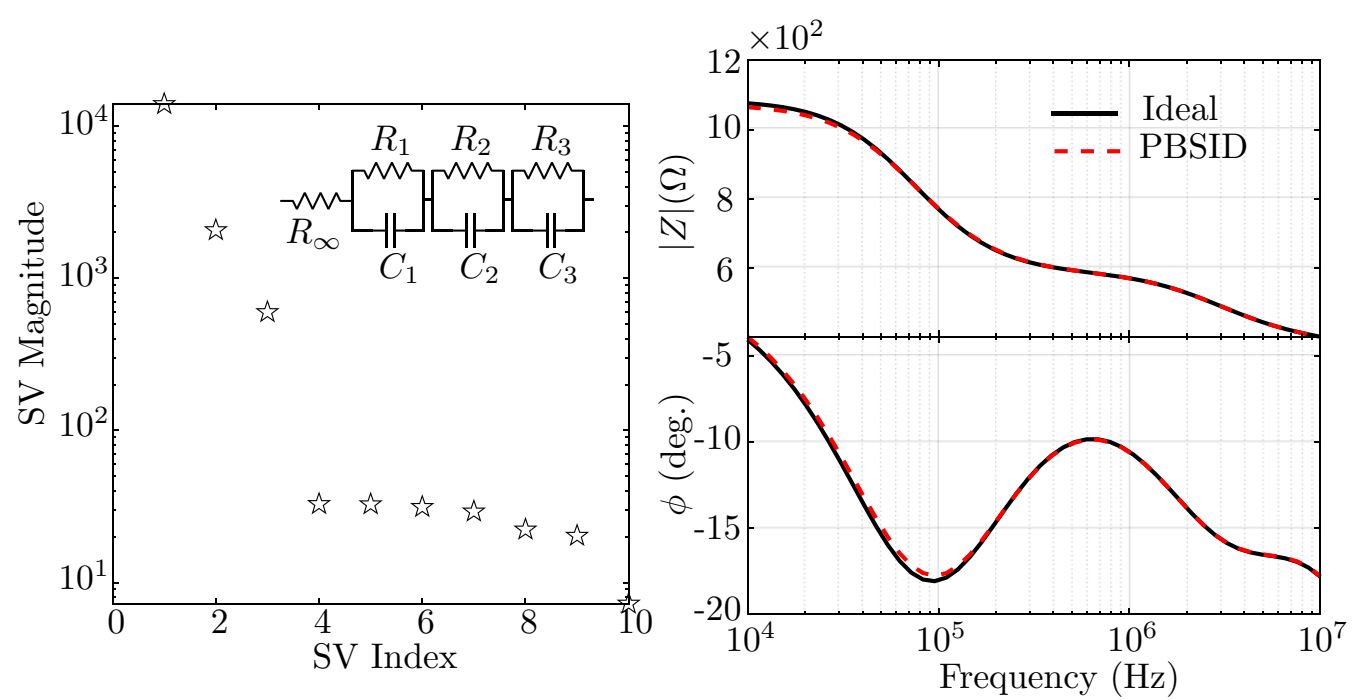

Figure 6. Subspace modeling of a multiple-dispersion calibration network. (a) Singular value plot showing the estimated model order $\hat{n}=3$ using the PBSID-approach. Inset: structure of the third-order measured circuit. (b) FR magnitude and phase spectra in the range from $10 \mathrm{kHz}$ up to $10 \mathrm{MHz}$.

Table 2. Estimated parameters via PBSID modeling of a multiple dispersion circuit.

\begin{tabular}{lrrr}
\hline Parameter (units) & \multicolumn{1}{c}{$\theta_{\mathrm{T}}$} & \multicolumn{1}{c}{$\theta_{\mathrm{PBSID}} \pm s_{\mathrm{p}}$} & $\% \delta_{\mathrm{p}}$ \\
\hline$R_{\infty}(\Omega)$ & 50.26 & $50.20 \pm 0.18$ & 0.12 \\
$\tau_{1}(\mathrm{~ns})$ & 3.44 & $3.42 \pm 0.25$ & 0.58 \\
$C_{1}(\mathrm{pF})$ & 10.30 & $10.29 \pm 0.75$ & 0.09 \\
$R_{1}(\Omega)$ & 331.80 & $332.28 \pm 2.60$ & 0.14 \\
$\tau_{2}(\mathrm{~ns})$ & 58.47 & $58.46 \pm 0.78$ & 0.01 \\
$C_{2}(\mathrm{pF})$ & 295.60 & $292.14 \pm 4.06$ & 1.17 \\
$R_{2}(\Omega)$ & 199.76 & $200.13 \pm 0.04$ & 0.18 \\
$\tau_{3}(\mu \mathrm{s})$ & 2.44 & $2.40 \pm 0.26$ & 1.63 \\
$C_{3}(\mathrm{nF})$ & 4.80 & $4.82 \pm 0.18$ & 0.41 \\
$R_{3}(\Omega)$ & 503.17 & $498.80 \pm 5.63$ & 0.86 \\
\hline
\end{tabular}

was determined with a $\mathrm{VAF}_{k}=99.1 \%$. Then the SS representation was converted to the following $\mathrm{TF}$

$$
\hat{H}_{\mathrm{PBS}}(s ; \hat{\boldsymbol{\theta}})=\frac{\hat{b}_{\mathrm{DL}}}{s}+\frac{\hat{b}_{1}}{s+\hat{a}_{1}},
$$

where $\hat{\boldsymbol{\theta}}=\left[\begin{array}{lll}\hat{b}_{1} & \hat{a}_{1} & \hat{b}_{\mathrm{DL}}\end{array}\right]$ is the vector of estimated coefficients, and in turn, it is related to the equivalent circuit parameters as in (24). Owing to the electrical properties of PBS and the impedance measurement effects, the TF (29) is a secondorder model with an integrator modeling the double-layer capacitance, as well as one pole related to the time-constant provided by the conductive and dielectric phenomena of the electrolyte under study. Hence, it is straightforward to deduce that, the TF has the equivalent circuit structure as shown in the inset of figure 7(a). Next, we compute the FR of (29) in the range from $10 \mathrm{kHz}$ up to $10 \mathrm{MHz}$ according to (25). Figure 7(b) depicts the Bode plots for the experimental impedance data (dots), the EIS model (dashed line) and the FR of the PBSID model (continuous line). As expected, the PBSID well approaches experimental data and EIS with moderate discrepancy in the low-frequency magnitude and the high-frequency phase. Also, from figure 7(b), it is possible to highlight the following observations.

- In the low-frequency range, below $10^{5} \mathrm{~Hz}$, the straight-line in the magnitude and the phase value $\approx 90^{\circ}$ are related to capacitive effect due to the electrode-electrolyte interface, which shunts the PBS conductivity.

- In the high-frequency range, above $10^{5} \mathrm{~Hz}$, the electrical properties of PBS become dominant given by the change in phase values, and the drastic drop in the magnitude reaching a low resistance value.

To evaluate the model performance, we computed the $\mathrm{VAF}_{\omega}$ among the PBSID and EIS models, thus retrieving an accuracy of $99.3 \%$ for the magnitude and $97.1 \%$ for the phase. It confirmed that computing the FR of the PBSID-model is equivalent to the classical EIS measurement. To quantitatively asses the results, in table 3 we show the mean value of the estimated parameters with the data-driven model and their parametric uncertainty $\% s_{\mathrm{p}}$. Likewise, table 3 summarizes the values obtained by fitting EIS data to the proposed ECM, alongside with the parametric error $\% e_{\mathrm{p}}$ indicating the goodness of the fit. From table 3 one can see that both methods retrieved similar for the ECM parameters. Regarding the PBSID estimates, these show consistency by keeping an uncertainty below $1.0 \%$ for all parameters. On the other hand, the EIS method retrieved a maximum fitting error of $1.62 \%$ for the capacitor $C_{\mathrm{DL}}$. Although PBSID and EIS results are similar, the double layer capacitance $C_{\mathrm{DL}}$ showed a deviation $\% \delta_{\mathrm{p}}=3.69$, which is due to the non-ideal nature modeled by a CPE. Furthermore, it is worth to notice that the ECM parameters of table 3 can be related with the impedance spectra of figure 7(b). The double layer capacitance produces 


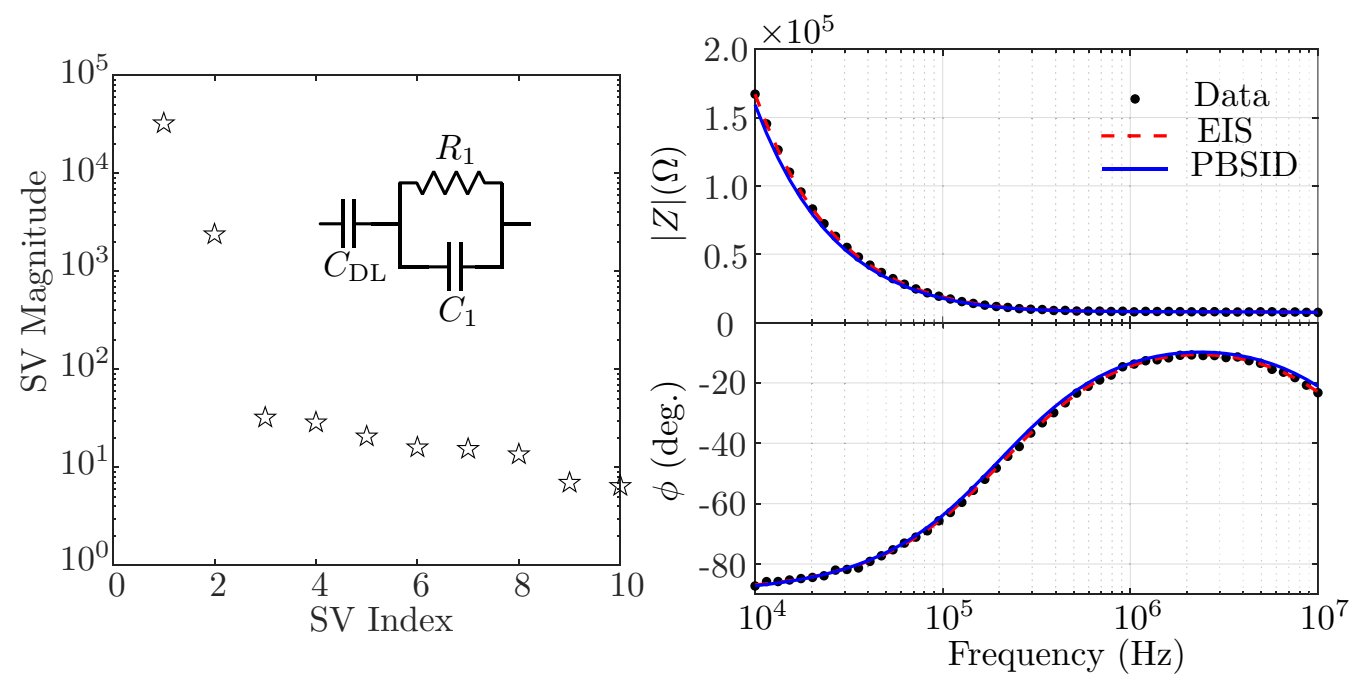

Figure 7. Subspace modeling of a phosphate buffer saline (PBS). (a) Singular value plot showing the estimated model order via PBSID. Inset: structure of the proposed equivalent circuit. (b) FR magnitude and phase spectra in the range from $10 \mathrm{kHz}$ up to $10 \mathrm{MHz}$.

Table 3. Estimated ECM parameters for $1 \mathrm{X}$ PBS solution using the PBSID scheme and the classical EIS method.

\begin{tabular}{lccllr}
\hline ECM Parameter (units) & $\theta_{\mathrm{PBSID}}$ & $\% s_{\mathrm{p}}$ & $\theta_{\mathrm{T}}$ & $\% e_{\mathrm{p}}$ & $\% \delta_{\mathrm{p}}$ \\
\hline$C_{\mathrm{DL}}(\mathrm{pF})^{\mathrm{a}}$ & 99.2 & 0.71 & 103 & 0.70 & 3.69 \\
$C_{1}(\mathrm{pF})$ & 0.73 & 0.23 & 0.74 & 0.93 & 1.35 \\
$R_{1}(\mathrm{k} \Omega)$ & 7.82 & 0.25 & 7.81 & 0.56 & 0.12 \\
$\tau_{1}(\mathrm{~ns})$ & 5.78 & 0.26 & 5.77 & 0.44 & 0.17 \\
\hline a & & &
\end{tabular}

${ }^{\mathrm{a}}$ For EIS $C_{\mathrm{DL}}$ is a CPE with $\alpha=0.9972$ (see equation (3)).

a large impedance in the low-frequency band. Meanwhile, the resistance of PBS, $R_{1}$, encodes its conductivity given by the flat magnitude response above $10^{5} \mathrm{~Hz}$. Also, the small capacitance $C_{1}$ relates the permittivity of the ionic solution as can be seen at frequencies above $10^{6} \mathrm{~Hz}$. With these results, we demonstrate how our method can derive a data-driven model for buffer solutions with a physical meaning.

4.2.2. Biological cells in suspension. The second experiment consisted in the measurement of a biological cell suspension to detect the underlying electrical processes. The sample was composed by cultured human keratinocyte cells of the HaCaT cell line. Briefly, the cells were cultured in Dulbecco's modified Eagles minimal essential medium. Afterwards, the cell culture was incubated at $37{ }^{\circ} \mathrm{C}$ and $5 \% \mathrm{CO}_{2}$ for $72 \mathrm{~h}$. Finally, the culture was washed and resuspended in PBS to further obtain a suspension of approximately 1000 cells. A $50 \mu \mathrm{l}$ volume of the PBS-HaCaT cells mixture was placed over the electrodes and measured as described in section 3 .

Figure 8(a) shows the SVD plot for the cell suspension, where the significant singular values are at the indices $\{1,2,3\}$, whose magnitude is at least two orders above the others singular values. Thus, the slot in the magnitude between the singular values 3 and 4, means that useful information of the cell suspension can be described with a SS model of order $\hat{n}=3$. In line with this finding, the accuracy of the model was determined with a $\mathrm{VAF}_{k}=98.7 \%$, such that the TF model describing the impedance of the cell suspension is

$$
\hat{H}_{\text {cells }}(s ; \hat{\boldsymbol{\theta}})=\frac{\hat{b}_{\mathrm{DL}}}{s}+\frac{\hat{b}_{1}}{s+\hat{a}_{1}}+\frac{\hat{b}_{2}}{s+\hat{a}_{2}}
$$

with $\hat{\boldsymbol{\theta}}=\left[\begin{array}{lllll}\hat{b}_{1} & \hat{a}_{2} & \hat{b}_{2} & \hat{a}_{1} & \hat{b}_{\mathrm{DL}}\end{array}\right]$ the parameters encoding the ECM structure. The TF (30) captures the dynamics of the cell suspension as follows. The integrator $\hat{b}_{\mathrm{DL}} / s$ models the double-layer capacitance of the electrode-PBS interface. Also, there are two first-order processes, each one modeling a resistive-capacitive effect in the sample. The first one could be related to the electrical properties of the PBS according with our previous findings; whereas, the second could be associated to the dielectric nature of the cells membrane. The ECM describing the cell suspension is shown in the inset of figure 8(a). In line with the PBSID scheme, we computed the FR of the model in (30), from $10 \mathrm{kHz}$ to $10 \mathrm{MHz}$ to compare it with the classical EIS method. In figure 8(b), we show the Bode plots for the experimental impedance data (dots), the EIS model (dashed line) and the FR of the PBSID model (continuous line). Therein, it is clear to see that the impedance magnitude $|Z|$ and the phase $\phi$ are comparable for both methods, with a maximum difference at the high-frequency for the phase values. Also, figure 8(b) confirms an adequate model structure given by the following remarks. 


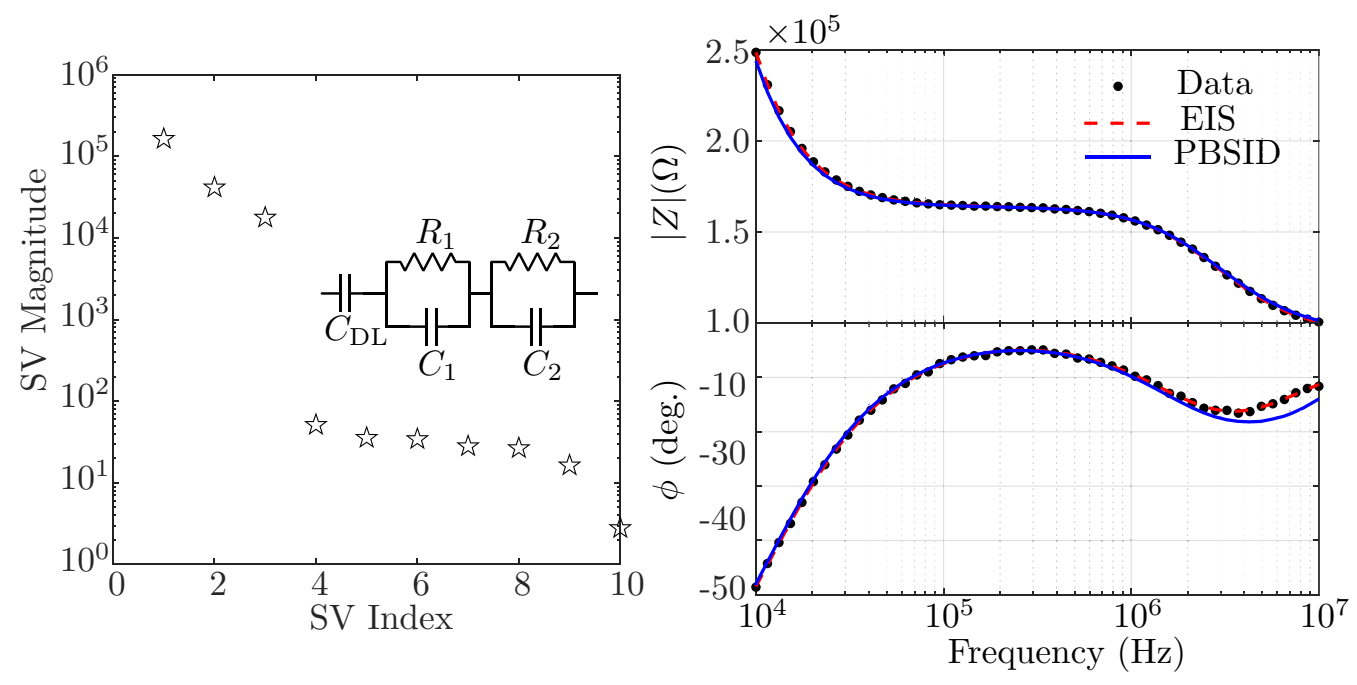

Figure 8. Subspace modeling of $\mathrm{HaCaT}$ biological cells in suspension. (a) Singular value plot showing the estimated model order via PBSID. Inset: structure of the proposed equivalent circuit. (b) FR magnitude and phase spectra in the range from $10 \mathrm{kHz} u p$ to $10 \mathrm{MHz}$.

Table 4. Estimated ECM parameters for HaCaT cells in suspension using the PBSID scheme and the classical EIS method.

\begin{tabular}{llllll}
\hline ECM Parameter (units) & $\theta_{\mathrm{PBSID}}$ & $\% s_{\mathrm{p}}$ & $\theta_{\mathrm{T}}$ & $\% e_{\mathrm{p}}$ & $\% \delta_{\mathrm{p}}$ \\
\hline$C_{\mathrm{DL}}(\mathrm{pF})^{\mathrm{a}}$ & 87.9 & 1.09 & 89.8 & 0.49 & 2.12 \\
$C_{1}(\mathrm{fF})$ & 3.56 & 2.39 & 4.57 & 0.54 & 22.1 \\
$R_{1}(\mathrm{k} \Omega)$ & 94.2 & 1.58 & 94.1 & 0.20 & 0.10 \\
$\tau_{1}(\mathrm{~ns})$ & 0.34 & 1.63 & 0.43 & 0.54 & 12.2 \\
$C_{2}(\mathrm{pF})$ & 0.88 & 0.34 & 0.89 & 0.92 & 1.14 \\
$R_{2}(\mathrm{k} \Omega)$ & 69.4 & 0.64 & 69.5 & 0.36 & 0.14 \\
$\tau_{2}(\mathrm{~ns})$ & 61.1 & 0.64 & 60.5 & 0.98 & 0.99 \\
\hline a For EIS, $C_{\mathrm{DL}}$ and $C_{1}$ are CPE with $\alpha=0.9842$ and $\alpha_{1}=0.7721$, respectively (see equation (3)).
\end{tabular}

- At frequencies below $10^{5} \mathrm{~Hz}$ the high magnitude and phase reflect the double-layer capacitance due to the electrodePBS interface.

- At frequencies within $10^{5}-10^{6} \mathrm{~Hz}$, the influence of the PBS conductivity is more evident as the impedance magnitude is flat and the phase tends to increase.

- At frequencies above $10^{6} \mathrm{~Hz}$, the dielectric effect of the cell membrane is reflected by an inflection point in the magnitude and a local minimum in the phase near to $4 \mathrm{MHz}$.

Taking the $\mathrm{VAF}_{\omega}$ as the metric of performance, the frequency-domain accuracy was $99.5 \%$ and $97.4 \%$ for the magnitude and phase, respectively. In table 4, we summarize the parameters retrieved by the PBSID model $\left(\theta_{\mathrm{PBSID}}\right)$ and with the classical EIS $\left(\theta_{\mathrm{T}}\right)$. For both cases, we show the performance analyses given by the parametric uncertainty $\% s_{\mathrm{p}}$ for PBSID, and the parametric error $\% e_{\mathrm{p}}$ for EIS fitting. Though the discrepancy $\% \delta_{\mathrm{p}}$ among the parameters is low for almost all cases, the capacitor $C_{1}$ and the time-constant $\tau_{1}$ have the largest deviation. This is due to $C_{1}$ is not an ideal capacitor and is better to model it with a CPE. Furthermore, the ECM parameters in table 4 have a close relationship with electrical processes of the cell suspension and the FR in figure $8(\mathrm{~b})$. The double layer is modeled by the capacitance $C_{\mathrm{DL}}$, conducting to high impedance values at the low-frequency band. On the other hand, the R-C parallel network, comprising $R_{1}$ and $C_{1}$, is modeling the presence of $\mathrm{HaCaT}$ cells in the sample, and is identified at frequencies above $10^{6} \mathrm{~Hz}$. This can be regarded as the effective capacitance and resistance describing the interfacial polarization among the cellular membrane and the surrounding medium. Ultimately, the second R-C circuit, with elements $R_{2}$ and $C_{2}$, describes the electrical properties of the buffer solution, i.e. PBS. This can be confirmed by the flat magnitude response in the intermediate frequency range, as well as by the effective value of the capacitance $C_{2}$, influenced by the PBS permittivity $\left(\epsilon_{\mathrm{PBS}}=78\right)$ which is three orders of magnitude greater than the HaCAT cells capacitance, $C_{1}$. According to this experiment, we show that the PBSID scheme is able to describe the electrical behavior of a cellular suspension, and it could be extended to further studies such as for monitoring cell growth and morphology, fouling and biophysical processes, to mention only a few.

\subsection{Benchmark test}

For comparison purposes, we contrasted the PBSID with the rule-of-thumb EIS for impedance biosensors. To this end, we selected eight scores: the measurement time $\left(t_{\text {meas }}\right)$, the time required for run the fitting algorithm $\left(t_{\text {run }}\right)$, if PK of the model structure is required, the optimization method for parameter 
Table 5. Benchmark score for PBSID and the classical EIS

\begin{tabular}{|c|c|c|c|c|c|c|c|c|}
\hline Method & $t_{\text {meas }}(\mathrm{ms})$ & $t_{\text {run }}(\mathrm{s})$ & PK & $\mathrm{OM}$ & $\# \theta^{\mathrm{a}}$ & $\mathrm{T} / \mathrm{F}$ & $\mathrm{CPE}$ & $\% \mathrm{~A}$ \\
\hline PBSID & $\sim 2.73$ & $\sim 0.72$ & No & Algebraic & $2(n)+2$ & Both & No & $>90 \%$ \\
\hline EIS & $\sim 12.46$ & $\sim 3.85$ & Yes & Iterative & $3(n)+3$ & $\mathrm{~F}$ & Yes & $>99 \%$ \\
\hline
\end{tabular}

${ }^{\mathrm{a}} n$ : Number of R-C circuits.

estimation, the number of parameters $(\# \theta)$ to estimate, if works in time- and frequency-domain $(\mathrm{T} / \mathrm{F})$, the ability to work with CPEs, and the accuracy of the method (\% A). Table 5 summarizes the performance of the PBSID and EIS methods.

From the benchmark test, one can notice that PBSID measures faster, has superior performance for signal processing, and is less computationally expensive than EIS. Though PBSID is a data-driven method working with algebraic methods for parameter estimation, its major drawback is that it cannot model CPE. This situation, indeed, currently limits the fitting accuracy of the PBSID method.

\section{Conclusion}

In this work, we introduced a data-driven scheme that automatically models the dynamics of impedance-based biosensors. The approach was based on the PBSID method working around the SVD of structured voltage and current signals. We showed how the subspace approach could estimate optimal ECMs without PK of the physical sample properties. The frequency-response of the derived data-driven models was comparable with classical EIS experiments from $10 \mathrm{kHz}$ up to $10 \mathrm{MHz}$, thus demonstrating the versatility of our approach. Due to PBSID cannot model CPEs, the proposed approach cannot compete in accuracy with the classical EIS methods. However, the obtained accuracy is acceptable to model dielectric phenomena of biological media for frequencies above tens of $\mathrm{kHz}$. We expect to improve the PBSID for modeling non-ideal circuit elements so the accuracy could be enhanced. Thereby, it will be possible to broaden the applications of the PBSID to model low-frequency phenomena, such as the Warburg impedance.

\section{Data availability statement}

All data that support the findings of this study are included within the article (and any supplementary files).

\section{Acknowledgment}

This work was supported by the Grant UNAM-PAPIIT TA100221.

\section{ORCID iDs}

Roberto G Ramírez-Chavarría (D) https://orcid.org/00000003-1825-4917
Maarten Schoukens (D) https://orcid.org/0000-0002-49041255

Luis Alvarez-Icaza (D) https://orcid.org/0000-0001-95163950

\section{References}

[1] Barsoukov E and Macdonald J R 2018 Impedance Spectroscopy: Theory, Experiment and Applications (New York: Wiley)

[2] Kanoun O 2018 Impedance Spectroscopy: Advanced Applications: Battery Research, Bioimpedance, System Design (Berlin: de Gruyter \& Co)

[3] Ramírez-Chavarría R G, Sánchez-Pérez C, Matatagui D, Qureshi N, Pérez-García A and Hernández-Ruíz J 2018 Ex-vivo biological tissue differentiation by the distribution of relaxation times method applied to electrical impedance spectroscopy Electrochim. Acta 276 214-22

[4] Spencer D C, Paton T F, Mulroney K T, Inglis T J J, Sutton J M and Morgan H 2020 A fast impedance-based antimicrobial susceptibility test Nat. Commun. 115328

[5] Xu T et al 2020 Characterization of red blood cell microcirculatory parameters using a bioimpedance microfluidic device Sci. Rep. 109869

[6] Chai C and Oh S 2020 Electrochemical impedimetric biosensors for food safety Food Sci. Biotechnol. 29 879-87

[7] Saini K, Kaushal A, Gupta S and Kumar D 2020 PlcA-based nanofabricated electrochemical DNA biosensor for the detection of Listeria monocytogenes in raw milk samples 3 Biotech 10327

[8] Naranjo-Hernández D, Reina-Tosina J and Min M 2019 Fundamentals, recent advances and future challenges in bioimpedance devices for healthcare applications J. Sens. 20199210258

[9] Ciucci F, Carraro T, Chueh W C and Lai W 2011 Reducing error and measurement time in impedance spectroscopy using model based optimal experimental design Electrochim. Acta 56 5416-34

[10] García E, Pérez P, Olmo A, Díaz R, Huertas G and Yúfera A 2019 Data-analytics modeling of electrical impedance measurements for cell culture monitoring Sensors 194639

[11] Al-Ali A, Elwakil A, Maundy B and Westwick D 2020 A generic impedance modeling technique AEU-Int. J. Electron. Commun. 123153301

[12] Simić M, Babić Z, Risojević V and Stojanović G M 2020 Non-iterative parameter estimation of the $2 \mathrm{R}-1 \mathrm{C}$ model suitable for low-cost embedded hardware Front. Inf. Technol. Electron. Eng. 21 476-90

[13] Xu Y, Li C, Jiang Y, Guo M, Yang Y, Yang Y and Yu H 2020 Electrochemical impedance spectroscopic detection of $\mathrm{E}$. coli with machine learning J. Electrochem. Soc. 167047508

[14] Ramírez-Chavarría R G, Sánchez-Pérez C, Romero-Ornelas L and Ramón-Gallegos E 2021 Time-constant-domain spectroscopy: an impedance-based method for sensing biological cells in suspension IEEE Sens. J. 21 185-92 
[15] Baronas R, Ivanauskas F and Kulys J 2021 Mathematical Models of Biosensors (Berlin: Springer)

[16] Boukamp B A 1995 A linear Kronig-Kramers transform test for immittance data validation J. Electrochem. Soc. 142 1885-94

[17] Zappen H, Ringbeck F and Sauer D U 2018 Application of time-resolved multi-sine impedance spectroscopy for lithium-ion battery characterization Batteries 464

[18] Ramírez-Chavarría R G, Quintana-Carapia G, Müller M I, Mattila R, Matatagui D and Sánchez-Pérez C 2018 Bioimpedance parameter estimation using fast spectral measurements and regularization IFAC-PapersOnLine $51521-6$

[19] Akulov S A, Fedotov A A, Akulova A S and Reshetnikova M A 2017 State space modeling of bioimpedance for haematocrit measurement 16th Int. Conf. on Biomedical Engineering ed J Goh, C T Lim and H L Leo (Singapore: Springer) pp 95-8

[20] Ivanisevic N, Rodriguez S and Rusu A 2019 Impedance spectroscopy based on linear system identification IEEE Trans. Biomed. Circuits Syst. 13 396-402

[21] Ramírez-Chavarría R G, Müller M I, Mattila R, Quintana-Carapia G and Sánchez-Pérez C 2019 A framework for high-resolution frequency response measurement and parameter estimation in microscale impedance applications Measurement 148106913

[22] Al-Ali A A, Elwakil A S, Maundy B J and Freeborn T J 2018 Extraction of phase information from magnitude-only bio-impedance measurements using a modified Kramers-Kronig transform Circuits Syst. Signal Process. $373635-50$

[23] Stupin D D, Kuzina E A, Abelit A A, Emelyanov A K, Nikolaev D M, Ryazantsev M N, Koniakhin S V and Dubina M V 2021 Bioimpedance spectroscopy: basics and applications ACS Biomater. Sci. Eng. 7 1962-86

[24] Raicu V and Feldman Y 2015 Dielectric Relaxation in Biological Systems: Physical Principles, Methods and Applications (Oxford: Oxford University Press)

[25] Dak P, Ebrahimi A and Alam M A 2014 Non-faradaic impedance characterization of an evaporating droplet for microfluidic and biosensing applications Lab Chip $142469-79$

[26] Xu Y, Xie X, Duan Y, Wang L, Cheng Z and Cheng J 2016 A review of impedance measurements of whole cells Biosens. Bioelectron. 77 824-36

[27] Retter U and Lohse H 2010 Electrochemical Impedance Spectroscopy (Berlin: Springer) pp 159-77

[28] Grossi M and Riccò B 2017 Electrical impedance spectroscopy (EIS) for biological analysis and food characterization: a review J. Sens. Sens. Syst.

$6303-25$

[29] Kaatze U and Feldman Y 2005 Broadband dielectric spectrometry of liquids and biosystems Meas. Sci. Technol. 17 R17-R35

[30] Joe Qin S 2006 An overview of subspace identification Comput. Chem. Eng. 30 1502-13

[31] Houtzager I, van Wingerden J and Verhaegen M 2009 VARMAX-based closed-loop subspace model identification Proc. 48th Conf. on Decision and Control (CDC)

[32] Houtzager I, van Wingerden J and Verhaegen M 2012 Recursive predictor-based subspace identification with application to the real-time closed-loop tracking of flutter IEEE Trans. Control Syst. Technol. 20 934-49

[33] Chiuso A 2007 The role of vector autoregressive modeling in predictor-based subspace identification Automatica 43 1034-48

[34] Yanai H, Takeuchi K and Takane Y 2011 Singular Value Decomposition (SVD) (New York: Springer) pp 125-49

[35] Gavish M and Donoho D L 2014 The optimal hard threshold for singular values is $4 / \sqrt{3}$ IEEE Trans. Inf. Theory $605040-53$

[36] Davis J H 2002 State Space Realizations (Boston, MA: Birkhäuser) pp 1-69

[37] Yuz J I and Goodwin G C 2013 Sampled-Data Models for Linear and Nonlinear Systems (Communications and Control Engineering) (London: Springer)

[38] Žic M, Subotić V, Pereverzyev S and Fajfar I 2020 Solving CNLS problems using Levenberg-Marquardt algorithm: a new fitting strategy combining limits and a symbolic Jacobian matrix J. Electroanal. Chem. 866114171

[39] Jiang Z, Yao J, Wang L, Wu H, Huang J, Zhao T and Takei M 2019 Development of a portable electrochemical impedance spectroscopy system for bio-detection IEEE Sens. J. $195979-87$

[40] Ljung L 1999 System Identification Theory for User 2nd edn (Englewood Cliffs, NJ: Prentice-Hall)

[41] Nasir M, Price D T, Shriver-Lake L C and Ligler F 2010 Effect of diffusion on impedance measurements in a hydrodynamic flow focusing sensor Lab Chip 10 2787-95

[42] Ma X, Du X, Li H, Cheng X and Hwang J C M 2018 Ultra-wideband impedance spectroscopy of a live biological cell IEEE Trans. Microw. Theory Techn. $663690-6$

[43] Verhaegen M and Verdult V 2007 Filtering and System Identification: A Least Squares Approach (Cambridge: Cambridge University Press) 